\title{
Genetic architecture of quantitative flower and leaf traits in a pair of sympatric sister species of Primulina
}

\author{
Chen Feng ${ }^{1,2} \cdot$ Chao Feng ${ }^{1} \cdot$ Lihua Yang ${ }^{1,2} \cdot$ Ming Kang $\mathbb{1}^{1,2} \cdot$ Mark D. Rausher $\mathbb{D}^{3}$
}

Received: 10 September 2018 / Revised: 19 November 2018 / Accepted: 19 November 2018 / Published online: 5 December 2018

(c) The Genetics Society 2018

\begin{abstract}
Flowers and leaves each represent suites of functionally interrelated traits that are often involved in species divergence and local adaptation. However, a major unresolved issue is how the individual component traits that make up a complex trait such as a flower evolve in a coordinated fashion to retain a high degree of functionality. We use a quantitative trait loci (QTL) approach to elucidate the genetic architecture of divergence in flower and leaf traits between the sister species Primulina depressa and Primulina danxiaensis, which grow sympatrically but in contrasting microhabitats. We found that flower traits were controlled by multiple QTL of small effect, while leaf physiological and morphological traits tended to be controlled by QTL of larger effect. The observed floral integration, manifested by a high degree overlap in both individual trait QTL and QTL for principal component scores (PCA QTL), may have been critical for evolutionary divergence of floral morphology in relation to their pollinators. This overlap suggests that direct selection on only one or a few of the component traits could have caused substantial divergence in other floral traits due to genetic correlations, while the low QTL overlap between floral and vegetative traits suggests that these trait suites are genetically unlinked and can evolve independently in response to different selective pressures corresponding to their distinct functions.
\end{abstract}

\section{Introduction}

Species divergence and local adaptation typically involve changes in suites of functionally interrelated traits such flowers and leaves. When floral structure diverges, as can occur when species or populations adapt to different sets of pollinators, many component traits such as corolla length and width, anther and stigma positions, and amount and concentration of nectar produced evolve simultaneously to

Electronic supplementary material The online version of this article (https://doi.org/10.1038/s41437-018-0170-2) contains supplementary material, which is available to authorized users.

$\triangle$ Ming Kang

mingkang@scbg.ac.cn

1 Key Laboratory of Plant Resources Conservation and Sustainable Utilization, South China Botanical Garden, Chinese Academy of Sciences, 510650 Guangzhou, China

2 University of Chinese Academy of Sciences, 100049 Beijing, China

3 Department of Biology, Duke University, Durham, NC 27708, USA produce an altered structure in which all of these component traits have the appropriate spatial relationships for facilitating efficient pollination by a specific set of pollinators (Berg 1960; Armbruster et al. 2009; Goodwillie et al. 2010). Similarly, adaptive divergence in leaves can involve modification of a suite of traits including leaf size, shape, agespan, cuticular properties, and physiological characteristics, such as chlorophyll, water, and nutrient content (Arntz and Delph 2001; Reich et al. 2003; Milla and Reich 2011). However, it is still poorly understood how the individual components of complex trait suites such as flowers and leaves evolve in a coordinated fashion to retain functionality.

Three scenarios may explain how coordinated evolution can occur. One extreme possibility is that genetic variation in each component trait is independent, and direct selection on each component trait, possibly coupled with correlational selection on groups of component traits, molds the final phenotype (Pigliucci 2003; Klingenberg 2008; Pélabon et al. 2014). A second possibility, at the other extreme, is that proper functional relationships among component traits may be assured by the genetic correlation structure, allowing direct selection on one or a small number of the component traits to result in indirect selection on the other 
component traits, essentially dragging them along in a manner that maintains their functional relationship (Armbruster et al. 2014). Studies of floral integration often report strong genetic correlations among floral component structure (Juenger et al. 2005; Bouck et al. 2007; Wessinger et al. 2014; Smith 2016), suggesting the possibility of this mechanism. However, it is not clear why it should be expected that the directionality of the correlation structure will be aligned to the direction in which selection attempts to move the population, especially if the structure of genetic correlations among component traits is largely determined by the mutational correlation structure (Lande 1980). A third possibility is that strong selection favoring change in one character leads to slightly maladaptive correlated changes in other characters, which are subsequently compensated for only slowly as appropriate mutations arise. Under this scenario, in recently diverged taxa, change in complex traits is the result of direct selection on a small number of component traits with the others diverging due to indirect selection. One way of distinguishing among these possibilities is to determine the extent to which mutations responsible for the evolution of component traits are pleiotropic.

In particular, if mutations generally affect only one or a small number of component traits, each trait or small group of traits will evolve independently; and if the changes are adaptive, they will require independent direct selection. By contrast, if mutations are highly pleiotropic with respect to the component traits, they will evolve in a coordinated fashion that could be driven by direct selection on one or a small number of the component traits. Previous studies on natural plant populations have revealed that phenotypic divergence can result from either a few quantitative trait loci (QTL) with large effect (Bradshaw et al. 1998; Bouck et al. 2007; Klahre et al. 2011) or many QTL of small effect (Goodwillie et al. 2006; Fishman et al. 2015; Wessinger et al. 2014). However, only a few studies have examined formally the extent to which the QTL for different characters overlap (Juenger et al. 2005; Hall et al. 2006; Lowry et al. 2015).

Others have used quantitative genetic breeding designs to examine the extent to which floral traits are genetically correlated (Edwards and Weinig 2011). These have generally found that floral traits tend to be correlated inter se, as do vegetative characters, but with little correlation between floral and vegetative characters. These results suggest that plant phenotypes may consist of more or less separated developmental modules, within which characters are highly correlated genetically, but between which correlations are weaker. However, genetic correlations arising due to segregating variation within individual species do not necessarily reflect pleiotropy of mutations that contribute to fixed differences between species, for two reasons: (1) within- species genetic correlations may be caused by linkage disequilibrium (Berg 1960; Armbruster and Schwaegerle 1996), and (2) fixed substitutions may reflect novel mutations that may have different pleiotropy characteristics than standing variation. Consequently, examining whether the same between-species QTL affects different traits is a more appropriate approach than examining within-species QTL for understanding the extent to which different traits have evolved independently.

When many correlated traits are examined, principal component analysis (PCA) can be used to reduce the dimensionality of the data, while retaining as much as possible of the variation in the original data (Jolliffe 1986; Upadyayula et al. 2006). This is achieved by identifying uncorrelated linear combinations of traits, the principal components (PCs), which are the eigenvectors of the phenotypic covariance matrix. Typically, a few leading PCs explain a large proportion of variance and can be considered new uncorrelated traits amenable to QTL detection (Jiang and Zeng 1995; Upadyayula et al. 2006). Reducing highly correlated variables into smaller sets of independent PCs through PCA may increase power of detecting QTL by reducing genome-wide false-positive detection rates.

In this study, we identify QTL contributing to differences in floral morphology and in leaf morphology and physiology between two closely related species of Primulina (Gesneriaceae) in southern China. This system is ideal for examining the genetic underpinnings of adaptive phenotypic divergence because of their sympatric distribution in different microhabitats. We quantify the degree to which QTL for different component traits overlap and test whether this overlap deviates from random expectations. In addition, we address the issue of the extent to which phenotypic divergence between these two species was actually caused by selection by applying a QTL sign test (QTLST; Orr 1998) to QTL for different traits. We use an $F_{2}$ population generated by a cross between one individual of each of these species from the sympatric site to identify QTL for both individual traits and PCs for a number of floral and leaf traits. With this information, we ask two questions: (1) to what extent do QTL for different traits overlap in genomic location? And (2) is there evidence indicating that divergent selection was responsible for differentiation of any of the traits?

\section{Materials and methods}

\section{Study system}

Primulina is a speciose genus of about 180 described species (Xu et al. 2017). This genus displays remarkable interspecific variation in flower and leaf traits and species 
occur in diverse light regimes, from steep outcrops exposed to full sunlight to dark caves with low light levels. Here we focus on a pair of sister species, Primulina danxiaensis and Primulina depressa, with marked differences in flower and leaf traits. $P$. danxiaensis was initially described as a member of the genus Chiritopsis and has small, light-yellow flowers and round leaves, while $P$. depressa has large and blue-purple flowers and narrow-long leaves (Fig. S1). Both species are endemic to northern Guangdong, South China. The distinct habitats in which they grow differ in a number of abiotic and biotic characteristics that could serve as selective agents to cause divergence, including differences in light availability, soil moisture and nutrient content, and the community of pollinators available (Zhou et al. 2010; Hao et al. 2015). The two species occur in sympatry ( $<100 \mathrm{~m}$ between populations) at a location on Danxia Mountain, a National Park and UNESCO World Heritage site, Guangdong, South China. At this location, the two species grow in different nearby habitats: $P$. danxiaensis tends to occupy caves with dim light conditions, whereas $P$. depressa tends to grow on outcrops with more sun (Fig. S1). Despite the contrasting morphologies, multiple nuclear DNA sequence data revealed that these two species are phylogenetically sister species (Kong et al. 2017). Individuals of the $P$. danxiaensis population sympatric with $P$. depressa are genetically more similar to P. depressa than they are to other conspecific populations (Kong et al. 2017), suggesting that there may be gene flow between the two species. Nevertheless, phenotypic differentiation in sympatry is maintained, presumably by selection favoring local adaptation to different microhabitat niches.

\section{Crossing and cultivation}

In 2011, we collected several adult individuals of $P$. depressa and $P$. danxiaensis from the sympatric populations in the Danxia Mountain National Park. The parent lines were maintained in a greenhouse at South China Botanical Garden (SCBG). In 2012, an $\mathrm{F}_{1}$ family including 21 progeny was generated from a cross between an individual of $P$. depressa (o, DXS02-17) and an individual of $P$. danxiaensis (우, DXS04-5). In 2013, we obtained an $F_{2}$ mapping population consisting of 201 individuals by selfing a single $F_{1}$ plant. After germination, all plants were transplanted into $8-\mathrm{cm}$ pots filled with about $200 \mathrm{ml}$ Klasmann substrate (Klasmann Deilmann, Geeste, Germany) and were grown on benches in a fully randomized design at the SCBG greenhouse under natural light. All $\mathrm{F}_{2}$ seeds were collected from September to October and sowed in next February. Plants were watered as needed and fertilized with Miracle-Gro Tep6 compound fertilizer (Scotts Miracle-Gro Company, OH, USA) weekly.

\section{Trait measurement}

Twelve traits were measured, including seven flower morphological traits (upper petal width, lower petal width, corolla width, corolla height, corolla length, pistil length, and stamen length; Fig. S2), two leaf size traits (leaf length and leaf width), and three leaf physiology traits (concentrations of chlorophyll a, chlorophyll b, and carotenoids). These traits were measured on $10 \mathrm{P}$. depressa individuals, $9 P$. danxiaensis individuals, $3 \mathrm{~F}_{1}$ hybrids, and $201 \mathrm{~F}_{2}$ individuals.

We measured flower morphological traits on the first three to five flowers per individual on the day the flower opened. First, we measured all the corolla traits using digital calipers. We then carefully dissected the corolla and measured the pistil and stamen length.

We scanned three mature and fully expanded leaves collected from each individual. ImageJ v.1.49 software (Abràmoff et al. 2004) was used to quantify leaf length and leaf width. The largest mature leaf of each individual was sampled to measure the maximum leaf length and width.

To measure the chlorophyll and carotenoid content, we extracted leaf pigments from the mid portion of fully expanded leaves with acetone and quantified content photometrically, using a multiscan spectrophotometer (Epoch Take3, Biotek Instruments, Winooski, VT, USA) at 440, 645, and $662 \mathrm{~nm}$. Concentrations per leaf area were calculated with the formula of Fadeel (1962): $C_{\mathrm{a}}=9.78 \times$ $E_{662}-0.99 \times E_{645} ; C_{\mathrm{b}}=21.4 \times E_{645}-4.65 \times E_{662} ;$ and $C_{\mathrm{c}}=$ $4.69 \times E_{440}-0.268 \times C_{(\mathrm{a}+\mathrm{b})}$, where $C_{\mathrm{a}}, C_{\mathrm{b}}$, and $C_{\mathrm{c}}$ indicate concentrations of chlorophyll a, chlorophyll b, and carotenoids, respectively.

\section{Assessing trait independence}

To assess trait independence, we used PCA to identify major axes of trait variation and covariation (Jaing and Zeng 1995). In the $F_{2}$ population, seven individuals did not flower, probably due to hybrid breakdown, and their floral traits were thus missing data. We performed PCA with Bayesian PCA (the "bpca" function of the R package pcaMethods; Stacklies et al. 2007), which can deal with missing values via imputation (i.e. mean substitution). The phenotypic data were standardized with the "scale" algorithm to eliminate the noise from magnitude of different phenotypic composition. We calculated correlations among PC scores and phenotypic traits to determine the relative contributions of different traits to each PC variable. PC scores were regarded as quantitative traits and used to identify QTL associated with these PCs. 


\section{Restriction-site associated DNA sequencing method (RAD-seq) genotyping by sequencing}

Genomic DNA was isolated from the fresh leaves of male and female parents, the $F_{1}$ hybrid that generated the $F_{2}$ population, as well as $201 \mathrm{~F}_{2}$ individuals using a modified CTAB-chloroform extraction protocol (Doyle and Doyle 1987) and was later quantified using Qubit fluorometer (Invitrogen, Carlsbad, CA, USA). To obtain markers for mapping, we prepared our DNA for a RAD-seq (Baird et al. 2008). In brief, DNA was digested with the restriction endonuclease EcoRI, which recognizes a 6-bp sequence (G'AATTC). Fragments with lengths about $350 \mathrm{bp}$ were separated on an agrose gel, selected for PCR amplification as sequencing templates, and sequenced on Illumina $\mathrm{XTen}{ }^{\mathrm{TM}}$ platform under the PE 150 protocol. The library construction and next-generation sequencing were performed by the staff of Novogene Bioinformatics Institute (Beijing, China).

Reads were quality-filtered by removing adapter sequences and reads containing $>10 \%$ low-quality bases with Q20 value $\leq 20 \%$. The quality filtration pipeline was provided by Feng et al. (2017). The quality-filtered RAD reads of each individual were mapped onto the Primulina huaijiensis genome (C. Feng, unpublished data) with the software BWA version 0.7.5 (Li and Durbin 2009). Based on the alignment result, Stacks software system version 1.44 (Catchen et al. 2011) was used to group homologous Illumina short reads into loci and determine individual genotypes. Putative haplotypes were built for each individual using a minimum stack depth parameter of five identical reads $(m=5)$. Identical loci from the two parents were then merged to build a catalog, with a maximum number of mismatches of three nucleotides $(n=3)$. Markers that were heterozygous in $F_{1}$ but homozygous in both parents were suitable for linkage mapping.

\section{Genetic map construction}

The Primulina RAD linkage map was constructed with the JoinMap v.4.0 program (van Ooijen 2006), using markers with a maximum of 20 missing genotypes out of 201 progeny. The segregation of each marker was analyzed by a chi-square $\left(\chi^{2}\right)$ test for goodness-of-fit to the expected Mendelian ratio 1:2:1 for biallelic codominant markers in an $\mathrm{F}_{2}$ population $(P<0.005)$. Markers were assigned to linkage groups (LGs) with an independence logarithm of odds (LOD) score of 7.0. The marker order within a LG was evaluated using default JoinMap v.4.0 parameters. Recombination rate was converted into genetic map distance (cM) by Kosambi's mapping function (Kosambi 1944).

\section{QTL mapping and analysis}

We adopted two approaches to assessing the extent to which QTL for different traits overlap. The first approach was to detect QTL for individual traits, while the second was to first conduct a PC analysis for all traits and then identify QTL for PC scores (PCA QTL). The latter approach may have the potential to be more powerful for detecting QTL's affecting multiple traits (Jiang and Zeng 1995). For both approaches, we used the interval mapping algorithm implemented in the MapQTL v.6.0 software using $1 \mathrm{cM}$ increments (van Ooijen 2004) to map both individual trait QTL and PCA QTL. A significance LOD threshold was determined for each LG using 10000 permutations to test for segregating QTL with a 5\% significance level. Markers with LOD scores exceeding the empirical threshold were first selected as cofactors before automatic cofactor selection was performed for each LG with a QTL. A multiple QTL-mapping analysis was finally performed using selected cofactors, and QTL were identified when LOD peaks were higher than the 5\% chromosome-wide LOD threshold determined by permutations. For each significant QTL, we obtained 1.5-LOD confidence intervals (CIs), as well as estimates of additive allelic effects. QTL effect sizes were reported as the proportion of phenotypic variation explained (PVE) and the proportion of the parental difference explained by the additive effects. Final QTL maps were drawn with the help of graphical package MapChart v.2.2 (Voorrips 2002).

In order to evaluate the significance of correspondence between QTL for different traits, we calculated the probability of obtaining the observed number of matching QTL by chance alone (Lin et al. 1995). This evaluation was determined by the given total length of genetic map and average interval length of all QTL, and the probability of overlap was estimated for different QTL intervals. To correct for multiple testing, we applied the Benjamini and Hochberg false discovery rate correction (Benjamini and Hochberg 1995) to determine which individual tests were significant at the overall $P=0.05$ level.

\section{QTL sign test}

We performed a QTLST (Orr 1998) to test for directional natural selection on both the individual trait and PCA QTL. This test examines the deviation from the null hypothesis that traits diverging via neutral processes (i.e. genetic drift) will accumulate alleles of both positive and negative effects, whereas traits evolving under directional selection will accumulate alleles with consistent effects. We preferred to implement QTLST rather than the more commonly used QTLEE (QTL equal effects) method because QTLST is less 
susceptible to ascertainment bias than QTLEE (Anderson and Slatkin 2003; Muir et al. 2014). For comparison, we also reported QTLEE test results, since many studies use this method. The log-normal distribution fitted the observed distribution of effect sizes in our study (data not shown), indicating that QTLST method was suitable to our QTL data (Orr 1998; Muir et al. 2014). We numerically integrated probability densities using the distr package (Ruckdeschel et al. 2006) and integrate function in R version 3.3.1 (R Development Core Team 2012), according to the method described by Muir et al. (2014). The code is available at Dryad.

\section{Results}

\section{Trait variation and correlations}

All 12 examined traits differed significantly between the two species (Table S1). PCA extracted four major PCs that accounted for a total of $89.4 \%$ of the variation among $\mathrm{F}_{2}$ individuals (Table 1). All measured traits and the four PCs exhibited continuous variation with unimodal distributions (Fig. 1 and Fig. S3). The first PC (PC1) explained $48.4 \%$ of the total trait variation with substantial positive loadings for all 12 variables, though loadings for flower morphological traits were somewhat stronger than for leaf traits (Table 1). We interpret this $\mathrm{PC}$ to represent variation in overall size. Because the other PCs are orthogonal to PC1, they can be

Table 1 Correlations between the first four principal component (PC) scores and each trait

\begin{tabular}{lcccc}
\hline Parameter & PC1 & PC2 & PC3 & PC4 \\
\hline Eigenvalue $(\lambda)$ & 5.79 & 2.45 & 1.59 & 0.86 \\
\% of total variation & 48.4 & 20.5 & 13.3 & 7.2 \\
Flower morphological traits & & & & \\
Upper petal width & 0.77 & -0.40 & -0.18 & 0.27 \\
Lower petal width & 0.82 & -0.36 & -0.17 & 0.29 \\
Corolla width & 0.81 & -0.14 & -0.10 & 0.08 \\
Corolla height & 0.75 & -0.37 & -0.18 & 0.36 \\
Corolla length & 0.90 & -0.16 & -0.01 & -0.30 \\
Pistil length & 0.83 & -0.10 & -0.06 & -0.38 \\
Stamen length & 0.77 & -0.11 & -0.03 & -0.54 \\
Leaf physiological trait & & & & \\
Concentration of chlorophyll a & 0.51 & 0.83 & -0.13 & 0.07 \\
Concentration of chlorophyll b & 0.53 & 0.78 & -0.11 & 0.12 \\
Concentration of carotenoids & 0.51 & 0.81 & -0.17 & 0.07 \\
Leaf size & & & & \\
Maximum leaf length & 0.43 & 0.07 & 0.86 & 0.10 \\
Maximum leaf width & 0.48 & 0.05 & 0.83 & 0.09 \\
\hline
\end{tabular}

Numbers are the percentage of total variation explained by each principal component
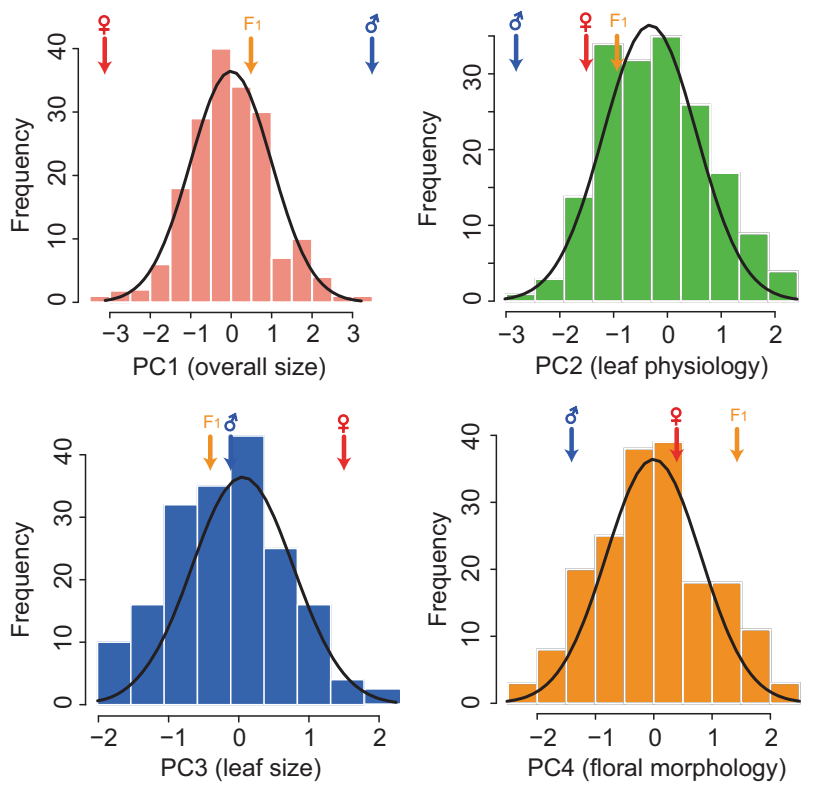

Fig. 1 Histograms of principal component scores for the four PCs in the $\mathrm{F}_{2}$ population. Mean values of Primulina depressa, P. danxiaensis, and $F_{1}$ hybrids are indicated with blue, red, and orange arrows, respectively

interpreted as explaining variance and covariance in subsets of traits independent of variation in overall size.

PC2 explained $20.5 \%$ of total variation with primary loadings $(0.78$ to 0.83$)$ for leaf physiological traits being positive, plus minor negative loadings $(-0.10$ to -0.40$)$ for flower size (Table 1). We interpret this PC as representing primarily variation in leaf physiological traits. PC3 explained $13.3 \%$ of total variation with substantial positive loadings ( 0.83 to 0.86 ) for leaf size, and thus represents leaf-size variation: large PC scores correspond to individuals that have large leaves relative to overall plant and flower size. Finally, PC4 explained $7.2 \%$ of total variation and displayed substantial loadings (0.08 to 0.54 ) for floral morphology, with petal width being positively and corolla length, pistil length, and stamen length being negatively correlated with PC score (Table 1). We consider this PC to represent primarily variation in floral morphology, indicating that individuals with high PC scores have shorter but wider flowers relative to overall plant size.

\section{Linkage mapping}

After quality filtering, a total of $14,313,717$ clean reads were obtained for the male parent (DXS02-17), 10,400,725 for the female parent (DXS04-5), and 21,566,255 for the $\mathrm{F}_{1}$ hybrid. The number of reads for the $201 \mathrm{~F}_{2}$ progeny ranged from 3,053,584 to 9,145,815 with an average of 4,680,666 (Supplementary Table S2). STACKS reconstructed 41,425 loci. Six individuals with genotypes for less than two thirds of loci were removed. We chose markers identified in at 


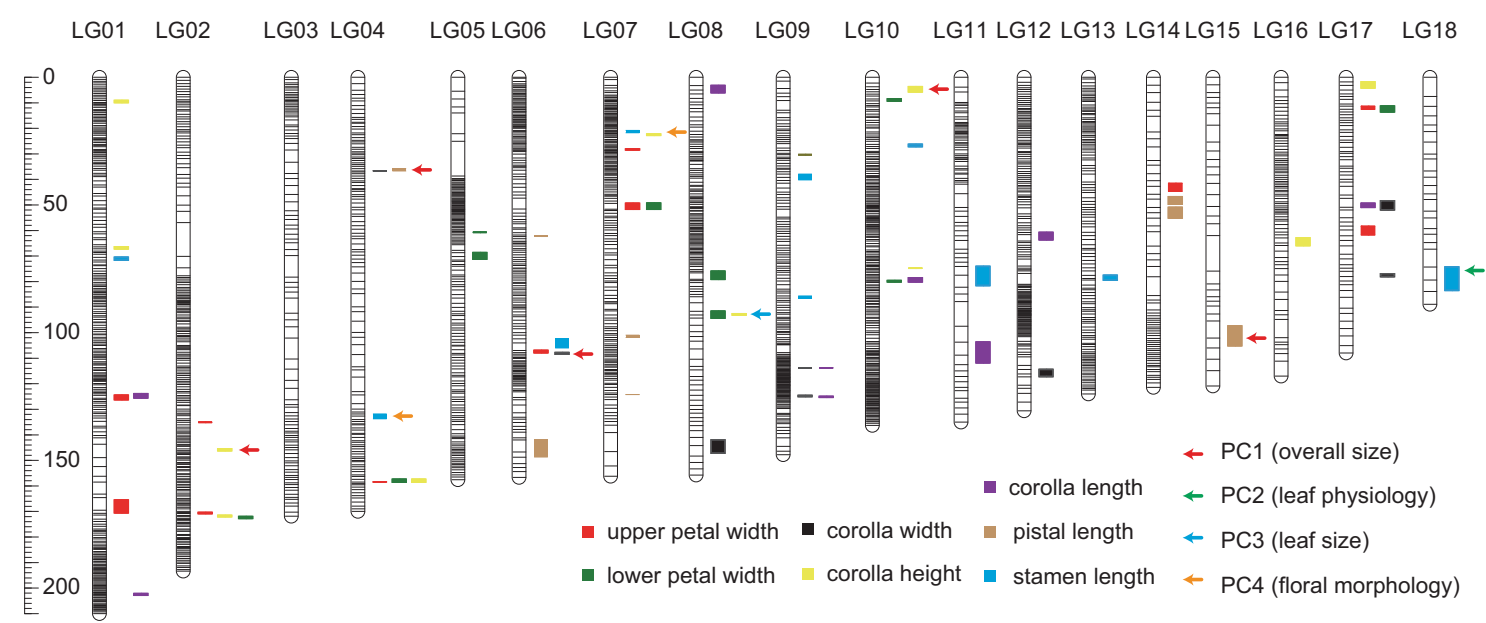

Fig. 2 Genome location of significant floral QTL. Confidence intervals of QTL are illustrated on the right side of the linkage groups. Arrows indicate co-localized PCA QTL

least $178 \mathrm{~F}_{2}$ individuals (90\% of 195 mappable individuals) and removed markers displaying significant segregation distortion at $P<0.005$ with JoinMap v.4.0. This led to a final 2534 mappable markers. Initial linkage mapping revealed that the inclusion of distorted markers (at $P<0.01$ ) did not affect the linkage arrangement compared with the map without distorted markers, and they were randomly distributed along the LGs with non-skewed markers. Therefore, these 831 segregation-distorted markers were included in the final linkage map, which led to a total of 2484 markers mapped on $195 \mathrm{~F}_{2}$ individuals. The final linkage map was assembled into 18 different LGs (Table S3 and Fig. 2), corresponding to the haploid number of chromosomes in P. depressa and P. danxiaensis $(n=18$, Kang et al. 2014).

The length of LGs ranged from 88.943 to $209.951 \mathrm{cM}$, for a total map length of $2601.510 \mathrm{cM}$ and an average distance between adjacent markers of $0.955 \mathrm{cM}$ (Supplementary Table S4). The number of markers on LGs ranged from 24 to 277 with an average of 138 markers. The minimum distance between adjacent markers was $0.017 \mathrm{cM}$ (LG08), and the maximum distance was $13.909 \mathrm{cM}$ (LG15; Supplementary Table S3).

\section{Analyses of individual trait QTL}

We identified a total of 114 QTL for the 12 individual phenotypic traits examined in this study. The number of QTL detected per trait ranged from 7 for leaf length to 11 for upper petal width, corolla height, and concentration of carotenoids (Table S5). PVE for the identified QTL ranged from $3.4 \%$ to $13.0 \%$, with the majority $(89.5 \%)$ of the QTL having small effects (PVE < 10\%; Table S5). These QTL accounted for $42.5-76.2 \%$ of the trait variation.
A total of 67 significant QTL was identified for flower morphological traits. The proportion of the parental difference explained by all QTL for these traits ranged from -0.18 to 0.84 , with a mean of 0.35 (Table S5). Generally, individual QTL for floral traits had small effects: none explained $>0.15$ of the parental difference, indicating that species differences in floral traits are highly polygenic.

In the 47 QTL for leaf traits, 30 were responsible for leaf physiological traits and 17 for leaf morphology. The proportion of the parental difference for leaf physiological and morphological traits explained by all QTL were 0.59 (range 0.49 to 0.74 ) and 0.005 (range -0.12 to 0.13 ), respectively (Table S5). Differences in leaf physiological traits were due primarily to QTL with moderate-to-major effect: $70 \%$ of the identified QTL explained $>15 \%$ of the parental difference, while $53 \%$ explained $>30 \%$ (Table S5). QTL effect sizes for leaf size traits were intermediate between those for floral and leaf physiological traits: $41 \%$ explained $>15 \%$ of the parental difference, while $18 \%$ explained $>30 \%$.

Of the 114 identified QTL, 37 (32\%) had overlapping 1.5-LOD confidence intervals with one to two other QTL from the same trait group while only nine QTL (8\%) overlapped with one other QTL from a different trait group (Table S5 and Figs. 2 and 3). Of the 21 trait pairs that exhibited QTL overlap, 11 pairs showed significantly more overlap than expected by chance ( 8 remain significant at the overall $P<0.05$ level after correction for false discovery rate, Table 2). Most of these ( 9 out of 11 , including the 8 that remain significant after false discovery rate correction) trait pairs were within groups, suggesting strong genetic integration due to pleiotropy within trait modules. In contrast, most trait pairs between categories (45 out of 53) showed no QTL overlap, and only 2 exhibited significantly more overlap than expected by chance. Neither of these remained significant after correction for false discovery rate. 


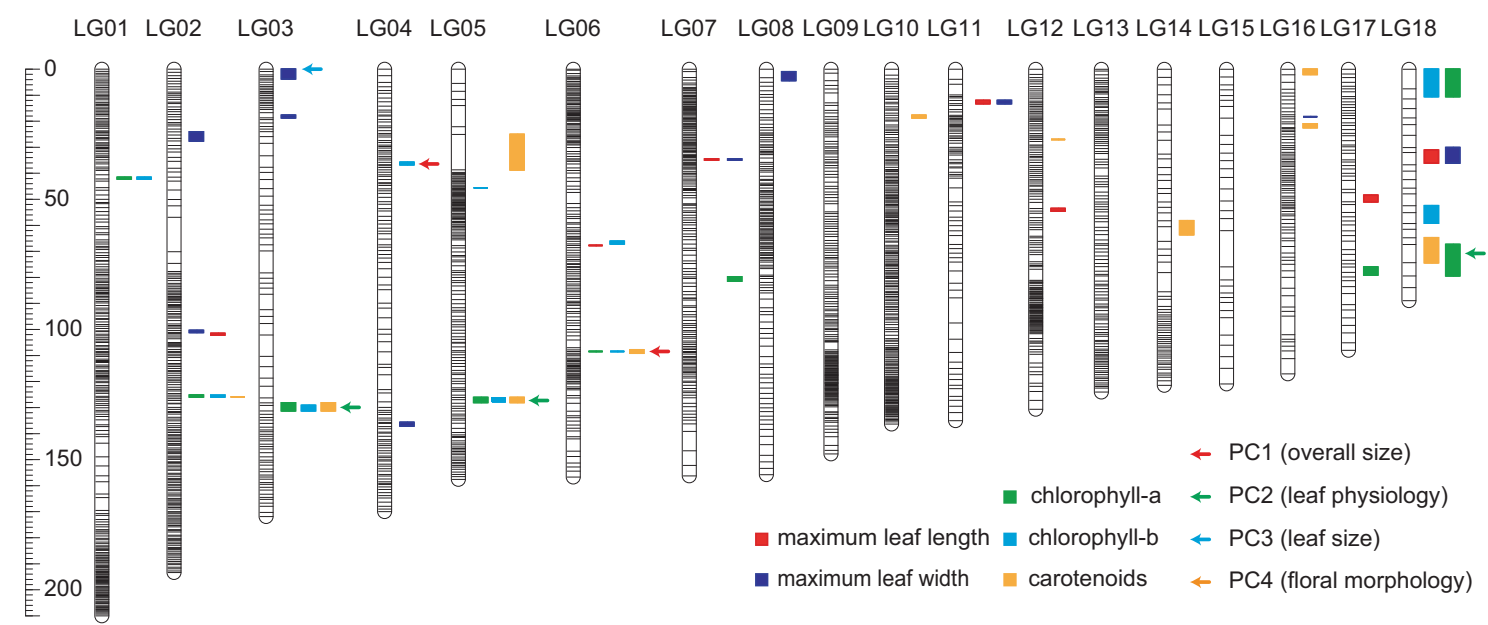

Fig. 3 Genome location of significant leaf QTL. Confidence intervals of QTL are illustrated on the right side of the linkage groups. Arrows indicate co-localized PCA QTL

\section{Analysis of PCA QTL}

We identified a total of 27 PCA QTL (Fig. 4, Table S5). Of these, eight corresponded PC1, six to PC2 (leaf physiology), six to PC3 (leaf size), and seven to PC4 (floral morphology). Nearly half (12) of the PCA QTL overlapped with individual trait QTL (Figs. 2, 3, and 4 and Table S5). Of the PC1 (overall size variation) QTL, three co-localized only with floral QTL (PC1_01,_06 and_07), while two colocalized with both floral traits and leaf physiology traits (PC1_02 and _04). Of the PC2 (leaf physiology) QTL, two co-localized with only leaf physiology QTL (PC2_01 and _02) and one with leaf physiology QTL and the stamen length QTL (PC2_06). For PC3 (leaf size) QTL, one overlapped with a leaf morphology QTL (PC3_01), while the other overlapped with floral morphology traits (PC3_03). Finally, two PC4 (floral morphology) QTL overlapped with floral QTL (PC4_03 and_06). Of the seven QTL that can be assigned to a specific module (e.g. PC2, PC3, PC4), six overlapped with trait QTL from the same module, while two overlapped with traits from a different module. Although the number of these PCA QTL is small, this pattern is similar to that seen in the individual trait QTL, in which substantially more co-localization occurs within modules than between modules.

Such a type of comparison is not possible for PC1, the overall size component, because it does not represent a single module. However, five PC1 QTL co-localize with individual trait QTL representing floral and leaf physiology traits, which suggests that these individual trait QTL reflect overall size variation rather than module-specific size variation. In addition, 15 PCA QTL did not overlap with individual trait QTL, and 97 individual trait QTL did not overlap with any PCA QTL (Table S5). These results suggest that two QTL approaches are complementary to each other.
In contrast with individual trait QTL, none of the PCA QTL overlap with each other (Fig. 4). This is perhaps not surprising given that the PCA components are orthogonal to each other, which would tend to prevent overlap among QTL from different PCs.

The effect sizes and total explained variation of the PCA QTL mirror those of individual trait QTL (Table S5). As for individual floral trait QTL, those associated with PC4 (floral morphology component) all explain $<10 \%$ of phenotypic variance in the trait, though $43 \%$ explain $>30 \%$ of the parental difference, suggesting that there are some QTL with moderate-to-large effect on these traits. In addition, all the PCA QTL together completely explain the parental difference for PC4. No QTL for PC2 (leaf physiology component) explain $>12 \%$ of variation, but many explain substantially larger portions of the parental difference (17\% $<0.15,50 \%>0.30$ ), paralleling the genetic architecture of individual leaf physiological traits. In addition, together all these QTL completely explain the parental difference. Finally, the PC associated with leaf size (PC3) appears intermediate, with all QTL explaining $<10 \%$ of the phenotypic variance, but $83 \%$ explaining $>0.15$ of the parental difference and $33 \%$ explaining $>0.3$. As with the individual leaf size traits, these PCA QTL together explained little of the parental difference in PC scores, presumably because alleles increasing the PC score are just about as common as alleles decreasing it.

All eight QTL associated with PC1 (overall size variation) individually were small, explaining $<10 \%$ of the parental difference. In addition, in sum they explained little of the parental difference because they differed in sign: five were individually negative, while three were individually positive. We note that, because general size does not represent one of the modules, we cannot compare these results with QTL from the modules. Nevertheless, this 
Table 2 Test for significance of QTL overlap between traits

\begin{tabular}{|c|c|c|c|c|c|c|c|c|c|c|c|c|}
\hline Trait & 1 & 2 & 3 & 4 & 5 & 6 & 7 & 8 & 9 & 10 & 11 & 12 \\
\hline \multicolumn{13}{|l|}{ 1. Upper petal width } \\
\hline $\begin{array}{l}\text { 2. Lower petal } \\
\text { width }\end{array}$ & $<0.001$ & & & & & & & & & & & \\
\hline 3. Corolla width & 0.076 & - & & & & & & & & & & \\
\hline 4. Corolla height & 0.078 & $<0.001$ & - & & & & & & & & & \\
\hline 5. Corolla length & $<0.001$ & 0.061 & $<0.001$ & - & & & & & & & & \\
\hline 6. Pistil length & - & - & 0.025 & - & - & & & & & & & \\
\hline 7. Stamen length & 0.093 & - & - & - & - & - & & & & & & \\
\hline $\begin{array}{l}\text { 8. Concentration of } \\
\text { chlorophyll a }\end{array}$ & 0.080 & - & - & - & - & - & 0.063 & & & & & \\
\hline $\begin{array}{l}\text { 9. Concentration of } \\
\text { chlorophyll b }\end{array}$ & - & - & 0.058 & - & - & 0.055 & - & $<0.001$ & & & & \\
\hline $\begin{array}{l}\text { 10. Concentration of } \\
\text { carotenoids }\end{array}$ & - & - & - & - & - & - & - & $<0.001$ & $<0.001$ & & & \\
\hline $\begin{array}{l}\text { 11. Maximum leaf } \\
\text { length }\end{array}$ & - & - & 0.039 & - & 0.048 & - & 0.056 & - & - & - & & \\
\hline $\begin{array}{l}\text { 12. Maximum leaf } \\
\text { width }\end{array}$ & - & - & - & - & 0.063 & - & - & - & - & - & 0.001 & \\
\hline
\end{tabular}

analysis indicates that the architecture of QTL affecting general size corresponds to many small-effect QTL. Given that floral traits load most strongly on PC1, this pattern may reflect our finding that individual floral QTL generally have small effect sizes.

\section{QTL sign test}

We found that most QTL (56 out of 67) of the individual flower traits were in the direction of divergence between the parent species (Supplementary Table S5), suggesting that directional selection for flower size might have played a role in trait divergence. This hypothesis was supported by Orr's QTLST implemented with QTLST and QTLEE, both of which showed that five flower traits (upper petal width, corolla width, corolla length, pistil length, stamen length) were likely to have diverged owing to natural selection (Table 3). In contrast, a substantial proportion of antagonistic QTL were detected for leaf traits, with average number of antagonistic QTL of 3.5 per trait (Supplementary Table S5). In addition, Orr's QTLST revealed no significant directional selection for these traits (Table 3).

We did not find significant directional selection for PCA QTL by either QTLST or QTLEE (Table 3). Results for PC2 (leaf physiology) and PC3 (leaf size) were consistent in this manner with tests performed on individual traits of the corresponding modules. However, in contrast with the individual trait analysis, for PC1 (overall size) and PC4 (floral morphology), QTL were inconsistent in sign and did not exhibit a signal of directional selection, even though they were highly or moderately correlated with the flower traits subjected to natural selection. We can offer no reason for this inconsistency, and thus this result should be interpreted with caution.

\section{Discussion}

\section{Trait modularity and divergence}

Our PCA of $\mathrm{F}_{2}$ phenotypes suggested that traits can be grouped into three modules, within which correlations between traits are high but between which correlations between traits are low. This pattern suggests that, during divergent evolution, these three modules evolve more or less independently, while traits within modules may evolve in a constrained, coordinated fashion. These inferences were largely confirmed by our QTL analyses. Analyses of both individual trait QTL and PCA QTL indicate that QTL overlap is substantially higher within modules than between modules.

Our tests for selection indicated only floral traits exhibited signatures of selection. Because of the low QTL overlap between floral traits and leaf traits, it is likely that selection on floral traits caused at most a small correlated response in leaf traits. The observed divergence in leaf traits was thus likely due either to independent selection on those traits or to genetic drift. Current data does not permit 
LG01 LG02 LG03 LG04 LG05 LG06 LG07 LG08 LG09 LG10 LG11 LG12 LG13 LG14 LG15 LG16 LG17 LG18
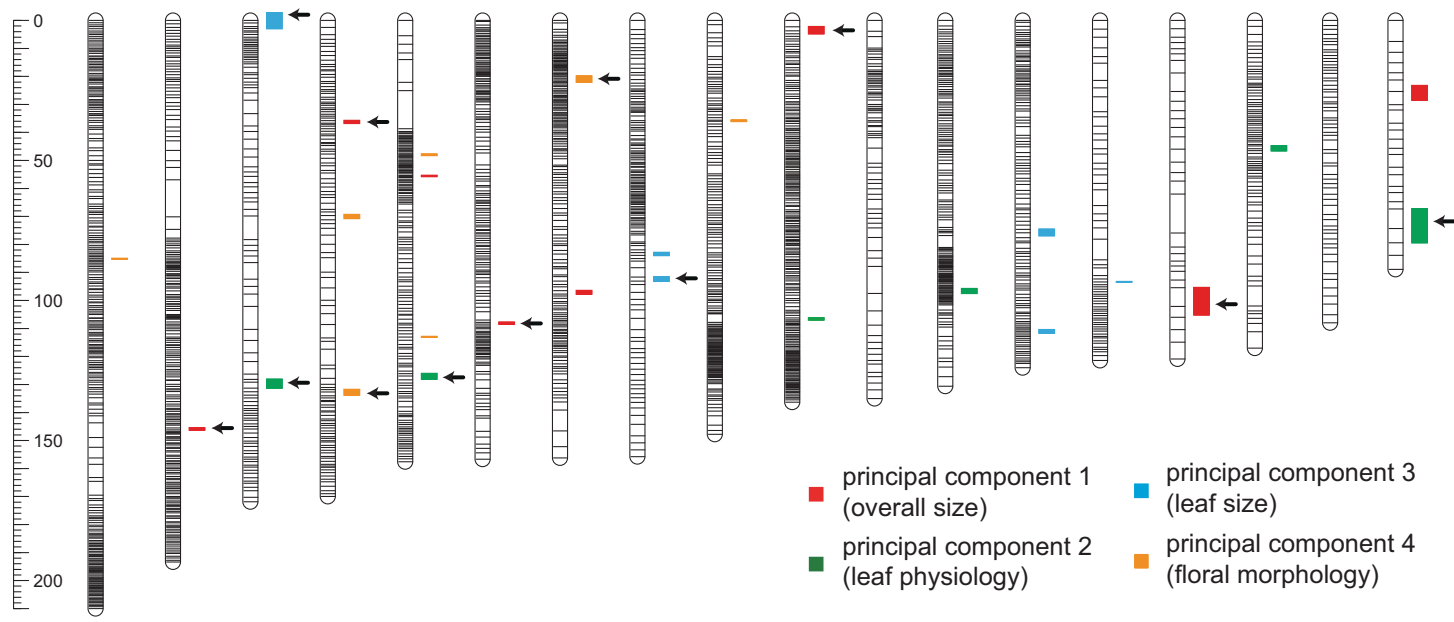

Fig. 4 Genome location of significant QTL for the first four PCs of phenotypic variation. Confidence intervals of QTL are illustrated on the right side of the linkage groups. Black arrows indicate regions that individual trait QTL co-localized

Table 3 QTLST and QTLEE results calculated from the number of QTL $(n)$, the number of QTL that altered a trait in the direction of Primulina depressa $(i)$, the phenotypic difference between species $(R)$, and the average absolute additive effect size of QTL $(|\bar{a}|)$

\begin{tabular}{lrrrrrr}
\hline Trait & \multicolumn{1}{c}{$i$} & $R$ & $|\bar{a}|$ & $P_{\mathrm{QTLST}}$ & $P_{\mathrm{QTLEE}}$ \\
\hline PC1 (total trait variation) & 8 & 3 & 0.82 & 0.23 & 0.995 & 0.885 \\
PC2 (leaf physiology) & 6 & 1 & -0.11 & 0.23 & 1.000 & 0.895 \\
PC3 (leaf size) & 6 & 3 & -0.23 & 0.21 & 0.882 & 0.737 \\
PC4 (floral morphology) & 7 & 1 & -0.26 & 0.23 & 1.000 & 0.932 \\
Upper petal width & 11 & 10 & 1.07 & 0.22 & $\mathbf{0 . 0 1 9}$ & $\mathbf{0 . 0 0 8}$ \\
Lower petal width & 10 & 7 & 1.07 & 0.56 & 0.368 & 0.208 \\
Corolla width & 8 & 3 & 0.91 & 0.31 & 0.994 & 0.887 \\
Corolla height & 11 & 10 & 1.22 & 0.66 & $\mathbf{0 . 0 1 4}$ & $\mathbf{0 . 0 0 8}$ \\
Corolla length & 9 & 9 & 1.59 & 0.76 & $\mathbf{0 . 0 0 5}$ & $\mathbf{0 . 0 0 4}$ \\
Pistil length & 8 & 8 & 1.45 & 0.95 & $\mathbf{0 . 0 0 9}$ & $\mathbf{0 . 0 0 6}$ \\
Stamen length & 10 & 9 & 1.39 & 0.50 & $\mathbf{0 . 0 2 9}$ & $\mathbf{0 . 0 1 7}$ \\
Concentration of & 9 & 7 & 0.91 & 0.97 & 0.190 & 0.120 \\
chlorophyll a & & & & & & \\
$\begin{array}{l}\text { Concentration of } \\
\text { chlorophyll b }\end{array}$ & 10 & 6 & 0.62 & 0.48 & 0.664 & 0.399 \\
Concentration of & 11 & 5 & 0.12 & 0.33 & 0.939 & 0.750 \\
carotenoids & & & & & & \\
Maximum leaf length & 7 & 1 & 0.99 & 0.18 & 1.000 & 1.000 \\
Maximum leaf width & 10 & 4 & -0.36 & 0.26 & 0.969 & 0.837 \\
\hline$P$
\end{tabular}

$P$ value is the probability that the empirical distribution of QTL signs would occur without directional natural selection, and significant $P$ values $(<0.05)$ are in bold

distinguishing between these possibilities. However, the apparent genetic independence of the three modules would suggest that, if divergent selection acted on either leaf physiology or leaf size, it would have done so largely independently on these two modules.

\section{Genetic architecture of flower and leaf trait divergence}

Our study suggests a highly polygenic genetic architecture involved in flower trait divergence between $P$. depressa and $P$. danxiaensis. QTL for individual traits generally explained small-to-moderate proportions $(<13 \%)$ of the phenotypic variance and a small proportion of the difference between parent means $(<0.15)$. A similar pattern was revealed by QTL for PC4 (floral morphology), although some of these had moderate-to-large effect $(>0.3$ of the parental difference explained). By contrast, for leaf physiological traits, individual QTL again explained small proportions of the phenotypic variance. However, in terms of proportion of parental difference explained, there were many individual QTL with moderate-to-large effect. QTL for PC2 (leaf physiology) exhibited a similar pattern. It would thus seem that species differences in these traits may be due primarily to QTL with large effect. Finally, the architecture of leaf size QTL fall somewhat between those of floral and leaf physiological traits: a mixture of QTL with small, intermediate, and large effects.

Our PCA allowed us to identify subsets of traits that corresponded to modules (PC2-leaf physiology, PC3leaf size, and PC4-flower morphology) and one axis that represented overall size (PC1). We detected eight QTL associated with PC1 that were generally of small effect size and had inconsistent directions. This suggests that divergence in overall size may have been largely due to genetic drift.

The effect sizes estimated and the number of QTL detected are likely to be influenced by a number of methodological factors, such as sample size and marker density 
(Beavis 1998). Despite the modest size of the mapping population $(N=195)$, identified QTL generally fell into narrow confidence intervals (Supplementary Table S5). Nevertheless, owing to modest sample sizes, the number of QTL resolved in this study is likely to be an underestimate of the actual number of genetic factors affecting trait divergence (Beavis 1998; Doerge 2002), thus resulting in an overestimation of the effect size of individual QTL. In addition, the identified QTL for each trait only accounted for ca. 43-76\% of variance (Supplementary Table S5), suggesting that a large number of QTL could have remained undetected. Despite these caveats, the possibility that trait divergence between the two species was affected by a few QTL of large effects can be ruled out definitively for floral traits. For leaf traits, QTL of large effect appear to be involved in addition to numerous QTL of small effect.

The highly polygenic genetic architecture of flower morphology in Primulina is comparable to that seen in many other plants. Similar results have been described for floral morphology in Mimulus (Fishman et al. 2002, 2015), Leptosiphon (Goodwillie et al. 2006), and Penstemon (Wessinger et al. 2014). However, our finding that many QTL of small effect underlie the flower trait differences between two related species that occur in sympatry contrasts with that observed by Ferris et al. (2017), who found that a few QTL of large effect underlie interspecific divergence in flower traits between two sympatric species of the Mimulus guttatus complex. They argue that their result reflects divergence in sympatry in the face of substantial gene flow, a situation in which divergence is expected theoretically to involve few loci with large effects, while mutations with small effect would be homogenized by gene flow (Yeaman and Whitlock 2011). In our case, gene flow between $P$. depressa and $P$. danxiaensis is likely minimal, because no morphologically intermediate individuals can be found in the field. This suggests that, given strong reproductive isolation, species can diverge in sympatry without largeeffect mutations.

Aspects of leaf morphology such as leaf size are often reported to be complex traits. Numerous studies have identified multiple QTL for leaf morphological traits for crop plants (Jiang et al. 2000; Frary et al. 2004; Welter et al. 2007; $\mathrm{Ku}$ et al. 2012); however, QTL underlying leaf morphological traits in natural populations have been less studied. In accordance with our results, a recent study in Mimulus found multiple small-to-moderate effect QTL for leaf morphology (Ferris et al. 2015). Leaf pigments play important roles in response to biotic and abiotic stresses, such as drought, wounding, cold temperatures, and pathogen infection (Mur et al. 2010; Guanter et al. 2014). Although the ecological significance of leaf pigments has been widely studied in diverse crops, considerably less is known about their genetic architecture in natural systems.
Our results indicate that difference in pigment contents between $P$. danxiaensis and $P$. depressa were dominated by substantial QTL with large effect, consistent with the observation in crops (Takai et al. 2010; Yin et al. 2015).

\section{Trait vs. PCA QTL}

Our study shows that standard QTL analysis on individual traits and analysis on PC scores provide complementary information. Each reveals QTL that the other does not, although some PCA QTL co-localize with some trait QTL. If one is interested in maximizing the number of QTL discovered, these results suggest that both types of QTL analysis should be conducted. In our case, we identified more trait QTL that did not co-localize with PCA QTL than PCA QTL that did not localize with trait QTL, but it seems likely that this pattern may be system specific and that in other species the opposite might be true. In any case, the patterns of variation revealed by the two approaches are very similar. In particular, both approaches indicate that QTL overlap is more common within modules than between modules. And both yield similar estimates of effect sizes and proportion of parental difference explained for the different modules.

\section{Selection, drift, and divergence}

QTLSTs showed that five flower traits (upper petal width, corolla width, corolla length, pistil length, and stamen length) were likely to have diverged owing to natural selection, suggesting that divergence in floral morphology was at least in part driven by selection. However, this test cannot distinguish between directional selection on individual traits and indirect selection due to genetic correlations with directly selected traits. The relatively high QTL overlap among these traits makes such correlated responses possible but does not rule out the possibility that selection acted on all five significant traits. However, for each floral trait, there is a substantial fraction of QTL that do not overlap with QTL of any other trait. If these diverged owing to selection, it follows that selection should have acted directly on those loci. Overall divergence in flower size thus may have involved a combination of response to direct selection and correlated responses to selection on other traits.

It is perhaps not surprising that our results indicate that floral morphology diverged owing to selection. Floral morphology influences mating system evolution and reproductive success, and different pollinators or different mating systems are likely to favor different morphologies. The evolution of selfing from outcrossing ancestors is often associated with changes that reduce flower size (Sicard and Lenhard 2011). In small isolated habitats where pollinators 
are rare, small-flowered genotypes capable of selfing can have a fitness advantage over larger outcrossing genotypes. Alternatively, divergent selection driven by adaptation to different pollinators may explain floral divergence. We observed that $P$. depressa is mainly visited by bees (Amegilla spp., Bombus spp., and Nomia spp.), while P. danxiaensis was visited by both bees (Nomia spp.) and hoverflies (Syrphidae) (L Yang, personal observations). It would therefore be of interest for future studies to assess selfing rates in the two sympatric Primulina species and to test the effectiveness of flower visitors as pollinators.

In contrast with floral trait QTL, we found no evidence for selection on QTL associated with PC4, which reflects floral trait variation. This result suggests that some of the QTL causing floral differences between the species have diverged owing to genetic drift rather than selection. Similarly, the prevalence of antagonistic effects in QTL for leaf traits, together with the nonsignificant QTLST for directional selection, suggest that these traits may really have diverged under weak selection or neutrality.

\section{Conclusions}

In this study, we have conducted QTL mapping of flower and leaf traits that differ between a pair of sympatric sister species of $P$. depressa and $P$. danxiaensis. We identified multiple QTL for each trait examined, suggesting a polygenic basis for trait divergence in this system. While individual QTL for floral traits have small effects, pigments and leaf size QTL tend to have large effect. The observed floral integration, manifested by a high degree overlap in floral trait QTL, may have been critical for evolutionary divergence of floral morphology in relation to their pollinators. This overlap suggests that direct selection on only one or a few of the component traits could have caused substantial divergence in other floral traits due to genetic correlations, while the lack of QTL overlap between floral and vegetative traits suggests that these trait suites are genetically unlinked and can evolve independently in response to different selective pressures corresponding to their distinct functions.

\section{Data archiving}

Raw Illumina sequences of RAD sequences have been deposited in NCBI sequence read archive (SRP148984). Genotypes and phenotype data for QTL analysis are available at the DRYAD repository: https://doi.org/10.5061/drya d. $49 \mathrm{t} 7030$.

Acknowledgements This work was supported by grant from the Natural Science Foundation of China (31570338) and Strategic
Priority Research Program of the Chinese Academy of Sciences (XDB31010000). We thank Marc Stift and three anonymous reviewers for their valuable comments on the manuscript.

\section{Compliance with ethical standards}

Conflict of interest The authors declare that they have no conflict of interest.

\section{References}

Abràmoff MD, Magalhães PJ, Ram SJ (2004) Image processing with ImageJ. Biophotonics Intern 11:36-42

Anderson EC, Slatkin M (2003) Orr's quantitative trait loci sign test under conditions of trait ascertainment. Genetics 165:445-446

Armbruster WS, Hansen TF, Pélabon C, Pérez-Barrales R, Maad J (2009) The adaptive accuracy of flowers: measurement and microevolutionary patterns. Ann Bot 103:1529-1545

Armbruster WS, Pelabon C, Bolstad GH, Hansen TF (2014) Integrated phenotypes: understanding trait covariation in plants and animals. Philos Trans R Soc B 369:20130245

Armbruster WS, Schwaegerle KE (1996) Causes of covariation of phenotypic traits among populations. J Evol Biol 9:261-276

Arntz AM, Delph LF (2001) Pattern and process: evidence for the evolution of photosynthetic traits in natural populations. Oecologia 127:455-467

Baird NA, Etter PD, Atwood TS, Currey M, Shiver AL, Lewis ZA et al. (2008) Rapid SNP discovery and genetic mapping using sequenced RAD markers. PLoS ONE 3:e3376

Beavis WD (1998) QTL analyses: power, precision, and accuracy. In: Paterson AH (ed). Molecular dissection of complex traits. CRC Press, New York, p 145-162

Benjamini Y, Hochberg Y (1995) Controlling the false discovery rate: a practical and powerful approach to multiple testing. J R Stat Soc B. https://doi.org/10.2307/2346101

Berg RL (1960) The ecological significance of correlation pleiades. Evolution 14:171-180

Bouck AC, Wessler SR, Arnold ML (2007) QTL analysis of floral traits in Louisiana iris hybrids. Evolution 61:2308-2319

Bradshaw HD, Otto KG, Frewen BE, McKay JK, Schemske DW (1998) Quantitative trait loci affecting differences in floral morphology between two species of Monkeyflower (Mimulus). Genetics 149:367-382

Catchen JM, Amores A, Hohenlohe P, Cresko W, Postlethwait JH (2011) Stacks: building and genotyping loci de novo from shortread sequences. G3 (Bethesda) 1:171-182

Doerge RW (2002) Mapping and analysis of quantitative trait loci in experimental populations. Nat Rev Genet 3:43-52

Doyle JJ, Doyle JL (1987) A rapid DNA isolation procedure from small quantities of fresh leaf tissue. Phytochem Bull 19:11-15

Edwards CE, Weinig C (2011) The quantitative-genetic and QTL architecture of trait integration and modularity in Brassica rapa across simulated seasonal settings. Heredity 106:661-667

Fadeel AA (1962) Location and properties of chloroplast and pigment determination in roots. Physiol Plant 15:130-147

Feng C, Xu M, Feng C, von Wettberg EJB, Kang M (2017) The complete chloroplast genome of Primulina and two novel strategies for development of high polymorphic loci for population genetic and phylogenetic studies. BMC Evol. Biol. 17:224

Ferris KG, Barnett LL, Blackman BK, Willis JH (2017) The genetic architecture of local adaptation and reproductive isolation in sympatry within the Mimulus guttatus species complex. Mol Ecol 26:208-224 
Ferris KG, Rushton T, Greenlee A, Toll K, Blackman BK, Willis JH (2015) Leaf shape evolution has a similar genetic architecture in three edaphic specialists within the Mimulus guttatus species complex. Ann Bot 116:213-223

Fishman L, Kelly AJ, Willis JH (2002) Minor quantitative trait loci underlie floral traits associated with mating system divergence in Mimulus. Evolution 56:2138-2155

Fishman L, Beardsley PM, Stathos A, Williams CF, Hill JP (2015) The genetic architecture of traits associated with the evolution of selfpollination in Mimulus. New Phytol 205:907-917

Frary A, Fritz LA, Tanksley SD (2004) A comparative study of the genetic bases of natural variation in tomato leaf, sepal, and petal morphology. Theor Appl Genet 109:523-533

Goodwillie C, Ritland C, Ritland K (2006) The genetic basis of floral traits associated with mating system evolution in Leptosiphon (Polemoniaceae): an analysis of quantitative trait loci. Evolution 60:491-504

Goodwillie C, Sargent R, Eckert CG, Elle E, Geber MA, Johnston MO et al. (2010) Correlated evolution of mating system and floral display traits in flowering plants and its implications for the distribution of mating system variation. New Phytol 185:311-321

Guanter L, Zhang Y, Jung M, Joiner J, Voigt M, Berry JA, Frankenberg C, Huete AR, Zarco-Tejada P, Lee JE (2014) Global and time-resolved monitoring of crop photosynthesis with chlorophyll fluorescence. Proc Natl Acad Sci 111:E1327-E1333

Hall MC, Basten C, Willis JH (2006) Pleiotropic quantitative trait loci contribute to population divergence in traits associated with lifehistory variation in Mimulus guttatus. Genetics 172:1829-1844

Hao Z, Kuang YW, Kang M (2015) Untangling the influence of phylogeny, soil and climate on leaf element concentrations in a biodiversity hotspot. Funct Ecol 59:165-176

Jiang C, Wright RJ, Woo SS, DelMonte TA, Paterson AH (2000) QTL analysis of leaf morphology in tetraploid Gossypium (cotton). Theor Appl Genet 100:409-418

Jiang CJ, Zeng ZB (1995) Multiple trait analysis of genetic mapping for quantitative trait loci. Genetics 140:1111-1127

Jolliffe IT (1986) Principle component analysis. Springer, New York

Juenger TJ, Pérez-Pérez M, Bernal S, Micol JL (2005) Quantitative trait loci mapping of floral and leaf morphology traits in Arabidopsis thaliana: evidence for modular genetic architecture. Evol Dev 7:259-271

Kang M, Tao JJ, Wang J, Ren C, Qi Q, Xiang Q et al. (2014) Adaptive and nonadaptive genome size evolution in Karst endemic flora of China. New Phytol 202:1371-1381

Klahre U, Gurba A, Hermann K, Saxenhofer M, Bossolini E, Guerin PM et al. (2011) Pollinator choice in petunia depends on two major genetic loci for floral scent production. Curr Biol 21:730-739

Klingenberg CP (2008) Morphological integration and developmental modularity. Ann Rev Ecol Evol Syst 39:115-132

Kong HH, Condamine FL, Harris AJ, Chen J, Pan B, Möller M et al. (2017) Both temperature fluctuations and East Asian monsoons have driven plant diversification in the karst ecosystems from southern China. Mol Ecol 26:6414-6429

Kosambi DD (1944) The estimation of map distances from recombination values. Ann Eugen 12:172-175

Ku LX, Zhang J, Guo SL, Liu HY, Zhao RF, Chen YH (2012) Integrated multiple population analysis of leaf architecture traits in maize (Zea mays L.). J Exp Bot 63:261-274

Lande R (1980) The genetic covariance between characters maintained by pleiotropic mutations. Genetics 94:203-215

Li H, Durbin R (2009) Fast and accurate short read alignment with Burrows-Wheeler transform. Bioinformatics 25:1754-1760

Lin YR, Schertz KF, Paterson AH (1995) Comparative analysis of QTLs affecting plant height and maturity across the Poaceae, in reference to an interspecific Sorghum population. Genetics 14:391-411

Lowry DB, Hernandez K, Taylor SH, Meyer E, Logan TL, Barry KW et al. (2015) The genetics of divergence and reproductive isolation between ecotypes of Panicum hallii. New Phytol 205:402-414

Milla R, Reich PB (2011) Multi-trait interactions, not phylogeny, finetune leaf size reduction with increasing altitude. Ann Bot 107:455-465

Muir CD, Pease JB, Moyle LC (2014) Quantitative genetic analysis indicates natural selection on leaf phenotypes across wild tomato species (Solanum sect. Lycopersicon; Solanaceae). Genetics 198:1629-1643

Mur LAJ, Aubry S, Mondhe M, Kingston-Smith A, Gallagher J, Timms-Taravella E, James C, Papp I, Hörtensteiner S, Thomas H, Ougham H (2010) Accumulation of chlorophyll catabolites photosensitizes the hypersensitive response elicited by Pseudomonas syringae in Arabidopsis. New Phytol 188:161-174

Orr HA (1998) Testing natural selection vs. genetic drift in phenotypic evolution using quantitative trait locus data. Genetics 149:2099-2104

Pélabon C, Firmat C, Bolstad GH, Voje KL, Houle D, Cassara J et al. (2014) Evolution of morphological allometry. Ann NY Acad Sci 1320:58-75

Pigliucci M (2003) Phenotypic integration: studying the ecology and evolution of complex phenotypes. Ecol Lett 6:265-272

R Development Core Team (2012) R: A language and environment for statistical computing. R Foundation for Statistical Computing, Vienna

Reich PB, Wright IJ, Cavender-Bares J, Craine JM, Oleksyn J, Westoby $\mathrm{M}$ et al. (2003) The evolution of plant functional variation: traits, spectra, and strategies. Int J Plant Sci 164:143-164

Ruckdeschel P, Kohl M, Stabla T, Camphausen F (2006) S4 classes for distributions. R News 6:2-6

Sicard A, Lenhard M (2011) The selfing syndrome: a model for studying the genetic and evolutionary basis of morphological adaptation in plants. Ann Bot 107:1433-1443

Smith SD (2016) Pleiotropy and the evolution of floral integration. New Phytol 209:80-85

Stacklies W, Redestig H, Scholz M, Walther D, Selbig J (2007) pcaMethods-a Bioconductor package providing PCA methods for incomplete data. Bioinformatics 23:1164-1167

Takai T, Kondo M, Yano M, Yamamoto (2010) A quantitative trait locus for chlorophyll content and its association with leaf photosynthesis in rice. Rice 3:172-180

Upadyayula N, Wassom J, Bohn MO, Rocheford TR (2006) Quantitative trait loci analysis of phenotypic traits and principal components of maize tassel inflorescence architecture. Theor Appl Genet 113:1395-1407

van Ooijen JW (2004) MapQTL ${ }^{\circledR} 5$ software for the mapping of quatitative trait loci in experimental populations. Kyazma, B. V., Wageningen, The Netherlands

van Ooijen JW (2006) JoinMap ${ }^{\circledR} 4$, software for the calculation of genetic linkage maps in experimental populations. Kyazma, B.V., Wageningen, The Netherlands

Voorrips R (2002) MapChart: software for the graphical presentation of linkage maps and QTLs. J Hered 93:77-78

Welter LJ, Göktürk-Baydar N, Akkurt M, Maul E, Eibach R, Töpfer R et al. (2007) Genetic mapping and localization of quantitative trait loci affecting fungal disease resistance and leaf morphology in grapevine (Vitis vinifera L). Mol Breed 20:359-374

Wessinger CA, Hileman LC, Rausher MD (2014) Identification of major quantitative trait loci underlying floral pollination syndrome divergence in Penstemon. Philos Trans R Soc B 369:20130349 
Xu WB, Guo J, Pan B, Zhang Q, Liu Y (2017) Diversity and distribution of Gesneriaceae in China. Guihaia 37:1219-1226

Yeaman S, Whitlock MC (2011) The genetic architecture of adaptation under migration-selection balance. Evolution 65:1897-1911

Yin Z, Qin Q, Wu F, Zhang J, Chen T, Sun Q, Zhang Y, Wang H, Deng D (2015) Quantitative trait locus mapping of chlorophyll a fluorescence parameters using a recombinant inbred line population in maize. Euphytica 205:25-35

Zhou Y, Wang X, Fu X, Zhou S, Li D (2010) Community features of rock vegetation of Danxia topography in Mount Jianglangshan, Zhejiang Province. J Zhejiang For Coll 27:699-705 